\title{
TIME STEPS V.S COHESION IN NON-SMOOTH CONTACT DYNAMICS ALGORITHM
}

\author{
L. STARON ${ }^{1}$ AND A. ABRAMIAN ${ }^{1}$ \\ ${ }^{1}$ Sorbonne Université, CNRS \\ Institut Jean Le Rond d'Alembert - UMR 7190, F-75005 Paris, France \\ lydie.staronesorbonne-universite.fr, anais.abramianeupmc.fr \\ http://www.lmm. jussieu.fr/ staron/
}

Key words: Granular Materials, Contact Dynamic Algorithm, Cohesion

\begin{abstract}
We present the equations obeyed by contacts forces in a granular system solved by the Contact Dynamics algorithm. We consider their resolution in a very simple case of cohesive interaction, i.e. for a straightforward two-body $2 \mathrm{D}$ normal collision. The equations predict that increasing time steps should coincide with an increase of the effective cohesion of the systems. Numerical simulations are performed to verify the predictions, in the case of cohesive granular piles falling in the gravity field. A discussion on how a seemingly purely numerical quantity may end up being a non-trivial ingredient in the physics of the simulated system ensues.
\end{abstract}

\section{INTRODUCTION}

One big challenge in modelling rigid discrete matter, even in its simplest form, lies in the inescapable existence of two distinct physical scales: the scale of the fragment, or grains, forming the media - their size, their typical displacement, their typical velocity...- and the scale of the contact. While the scale of the grains essentially obeys Newton's law of motion, the scale of the contact renders the physicochemical details of the contact mechanism, such as surface deformation, asperities, cohesion, friction, and so on. Beside the intrinsic physical complexity of contact phenomena, a major difficulty originates from the huge difference between the two characteristic times of each underlying physics: a microscopic tiny contact scale and a macroscopic (or mesoscopic) larger grain scale. This difficulty becomes very tangible when one undertakes to give a numerical description of the behaviour of a discrete granular media: the two different physical scales of contact and grains will translate into adapting a computational time step to account for the behaviour of the system. Logically, the smaller time step "wins", or the contact phenomena description would be lost. Some methods, known as "event-driven", mitigate the (hard) choice of a computational time scale by allowing the grains trajectories and collisions to dictate the times when computation is necessary [1]; these methods are however not suited to the modelling of dense static packings. Therefore, the most popular method, known as Discrete Element Method, explicitly describes the contact phenomena with a varying degree of details $[2,3,4]$. In the simplest case, as the spring and dashpot model, tiny time steps are necessary to account for the stiffness of the grains: the more rigid the grains, the tiniest the time steps [5]. The choice of the computational time step is closely linked to contact properties in DEM. It coincides with a complex inter-dependence between the physical elements 
describing the contact phenomena that are physically unconnected. Cohesion and stiffness are among those [6].

As an alternative, the Contact Dynamics (CD) method adopts the view that the details of the contact phenomena can be obliterated, as long as the rigidity of the grains, and the dissipative properties of the contacts, are rendered. Instead of treating contacts as complex microscopic phenomena, they appear in the evolution of the granular system in the shape of mathematical discontinuities [7, 8]. Thereby, the method gets rid of the physical time scale related to contacts. The computational time step is dictated by the macroscopic (grain-scale) dynamics, and does not interfere with the details of the contact laws. This seems reasonable for simple rigid grains for which contact forces obey a binary logic - being zero if grains do not touch and compressive if they do. The situation is less clear in the case of cohesive systems, for which tensile forces are permitted, and a cohesive threshold must be added to the contact non-smooth law. In that case, computational time steps do not systematically simplify out of the resolution.

In this paper, we write down the simple equations obeyed by contacts forces in Contact Dynamics algorithm. The minimalist case of a two-body cohesive collision is considered, and the stages of the contact force resolution are explained. We show how equations predict the increase of the effective cohesion of the system with the increase of the time step. To confirm this guess, numerical simulations are performed in the case of a small cohesive granular pile falling in the gravity field; predictions, and the role of the time step, are discussed.

\section{EQUATIONS: THE TWO-BODY CASE OF BINARY COLLISIONS}
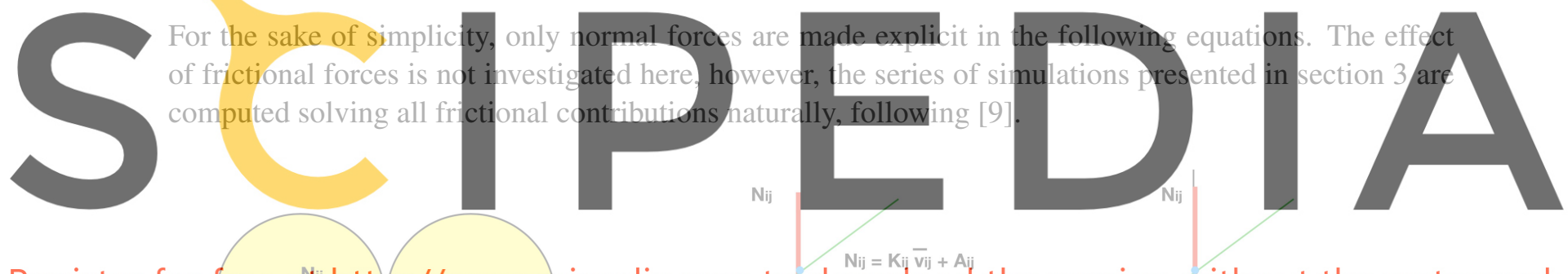

Register for free alti httos//www scipedia.com to $\mathrm{dow}_{\mathrm{Nij}}=\mathrm{Ki}_{\mathrm{ij}} \overline{\mathrm{V}}_{\mathrm{i}}+\mathrm{Aij}_{\mathrm{ij}}$

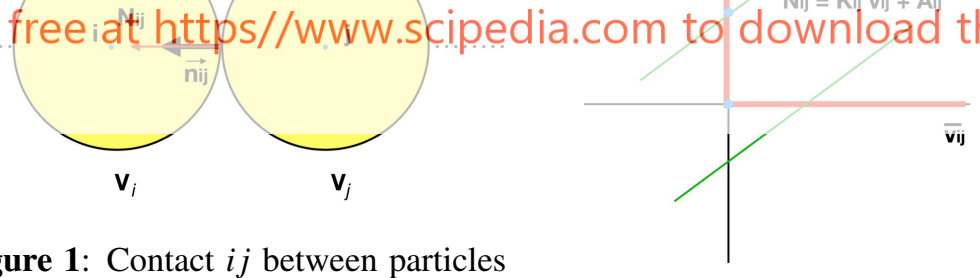

$i$ and $j$, transmitting the normale force $N_{i j}$ following the normal contact vector $\vec{n}_{i j}$, defining the contact's referential. The grains relative velocity at contact $v_{i j}=v_{i}-v_{j}$ (or contact velocity) is positive when the contact is opening, and negative when the contact is further closing.

a)

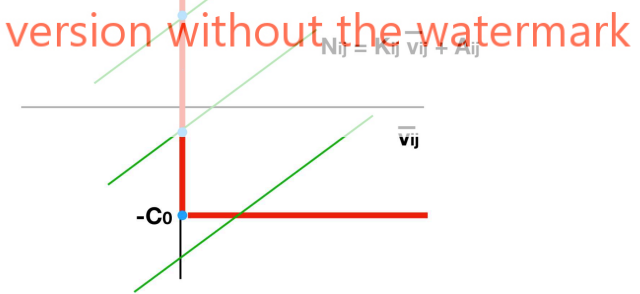

b)

Figure 2: Non-smooth contact graphs for the normal forces: forces are transmitted only at contact (no distant interaction) while respecting a hard core repulsion (no inter-penetration). Non-cohesive (a) and cohesive (b) cases are represented; $-C_{0}$ denotes the cohesive force threshold. In each case, all possible intersections with equation $N_{i j}=K_{i j} \bar{v}_{i j}+A_{i j}$ (5) are shown. Blue dots show the corresponding value of the force. 


\subsection{Generalities}

We consider the contact between the two grains $i$ and $j$ of mass $m_{i}$ and $m_{j}$ respectively, forming the contact $i j$ transmitting the normal force $N_{i j}$, as shown in Fig.1. The contact referential is chosen so that the normal contact vector $\vec{n}_{i j}$ is positive pointing towards $i$, and the force $N_{i j}$ is positive when compressive. We write for both grains the equations of dynamics, discretised over the time step $\Delta t$, in the contact referential $\vec{n}_{i j}$, with subscripts - and + denoting respectively the beginning and the end of the time step:

$$
\begin{aligned}
v_{i}^{+}-v_{i}^{-} & =\frac{\Delta t}{m_{i}} N_{i j} \\
v_{j}^{+}-v_{j}^{-} & =-\frac{\Delta t}{m_{j}} N_{i j}
\end{aligned}
$$

External forces such as gravity are not written here, and we consider only the contact force; writing them down would not change the outcome of the equations, but make the reading less clear.

Writing (1) - (2), we get:

$$
\begin{aligned}
\left(v_{i}^{+}-v_{i}^{-}\right)-\left(v_{j}^{+}-v_{j}^{-}\right) & =\Delta t\left(\frac{1}{m_{i}}+\frac{1}{m_{j}}\right) N_{i j} \\
v_{i j}^{+}-v_{i j}^{-} & =\frac{\Delta t}{m_{i j}} N_{i j}
\end{aligned}
$$
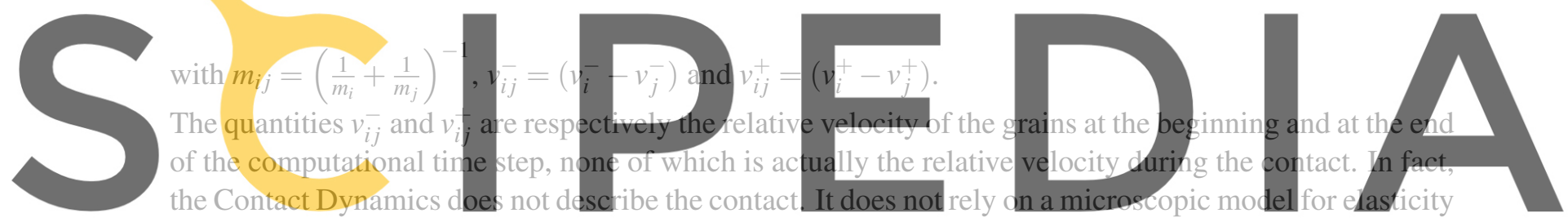

or tensile stress, and thus obliterates microscopic length scale. In this way, it disposes of microscopic

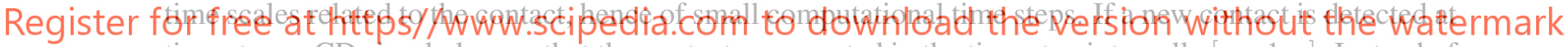

time step $n, C D$ simply knows that the contact was created in the time step intervalle $[n-1, n]$. Instead of

aiming at determining the exact grains relative velocity when the contact materialises, it does propose an

estimate for this contact velocity. Therefore, it introduces a formal velocity $\bar{v}_{i j}$ which is a combination of the velocities at the beginning and the end of the time step, weighted by the material coefficient of restitution, denoted $\rho$ in the following:

$$
\bar{v}_{i j}=\frac{v_{i j}^{+}+\rho v_{i j}^{-}}{1+\rho} .
$$

Rewritting (3) using (4) gives

$$
N_{i j}=+\frac{m_{i j}}{\Delta t}(1+\rho) \bar{v}_{i j}-\frac{m_{i j}}{\Delta t}(1+\rho) v_{i j}^{-}
$$

which we write

$$
N_{i j}=K_{i j} \bar{v}_{i j}+A_{i j}
$$

with $K_{i j}=\frac{m_{i j}}{\Delta t}(1+\rho)>0$, and

$$
A_{i j}=-\frac{m_{i j}}{\Delta t}(1+\rho) v_{i j}^{-} .
$$


As we see in equation (5), this straightforward formulation does not provide a unique solution for the contact force $N_{i j}$, but an infinity of mathematically possible $\left(N_{i j}, \bar{v}_{i j}\right)$ pairs. We hence need an additional constraint to determine the physically relevant pair: this constraint is given by the contact graphs (Fig.2).

\subsection{Solving normal forces using the hard-core repulsion non-smooth graph}

Contacts Dynamics relies on non-smooth hard-core repulsion graphs for solving normal forces, which provide an ensemble of possible solutions to be confronted with the contact force equation (5). The intersection of the two gives the $\left(N_{i j}, \bar{v}_{i j}\right)$ pair compatible with both Newton's law and hard-core repulsion (a similar technics is used for friction and tangential forces, but not presented here).

The non-cohesive non-smooth hard-core repulsion, known as Signorini's condition, ensures that forces take strictly positive values, only if the candidate grains for contact touch (in practice, numerically, if the grains overlap) (Fig 2-a). In the case of cohesive systems, a crude modification of the graph allows for negative values of the forces, that will cause the contact to withstand tensile forces and be cohesive (Fig 2-b).

Three cases can be distinguished, depending on where contact equation (5) and contact graph intersect.

2.2.1 Case 1: $A_{i j}>0$
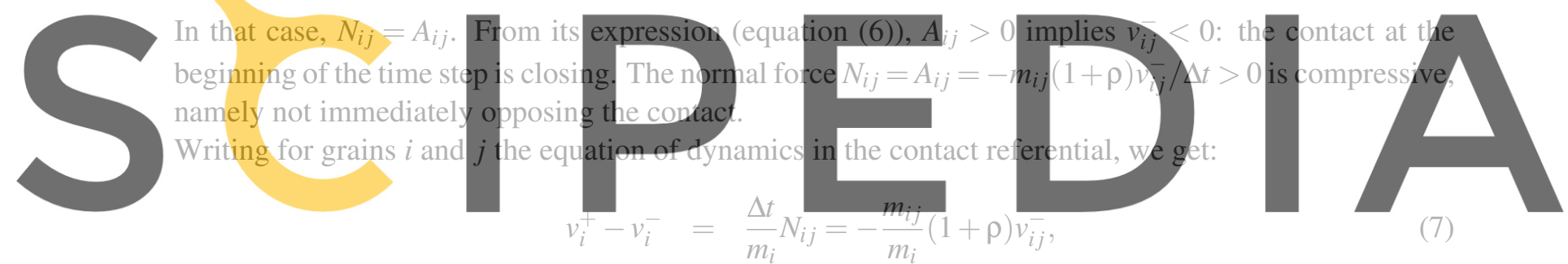

Register for free at https//www.scipedia.com
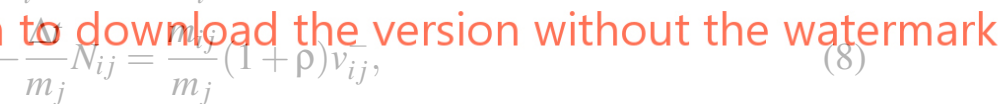

so that forming (7) - (8) readily gives:

$$
v_{i j}^{+}=-\rho v_{i j}^{-}
$$

We obtain the expression for a two-body collision with a restitution coefficient $\rho$. In our case, $v_{i j}^{-}<0$, which implies $v_{i j}^{+}>0$ : the contact, closing at the start of the time step, evolves towards opening at the end.

\subsubsection{Case 2: $-C_{0}<A_{i j}<0$}

In that case, as in case 1 (paragraph 2.2.1 above), CD prescribes $N_{i j}=A_{i j}$. The intersection in $A_{i j}<0$ implies $N_{i j}=-\frac{m_{i j}}{\Delta t}(1+\rho) v_{i j}^{-}<0$ is no longer compressive, but tensile. Accordingly $v_{i j}^{-}>0$ : the contact is opening at the start of the time step. Forming (7) - (8) readily gives $v_{i j}^{+}=-\rho v_{i j}^{-}$, namely the contact closes again at the end of the time step. In other words, the contact is allowed to out-live negative values 
of the contact force, as long as it is greater than the threshold $-C_{0}$. The contact withstands tensile forces and reverse from opening to closing again.

\subsubsection{Case 3: $A_{i j}<-C_{0}$}

In that proscribed condition, the intersection between the contact equation (5) and the contact graph corresponds to a force beyond the cohesive threshold $-C_{0}$. In that case, the contact graph imposes $N_{i j}=-C_{0}$, which implies, rewriting (7) - (8):

$$
v_{i j}^{+}=v_{i j}^{-}-\frac{\Delta t}{m_{i j}} C_{0} .
$$

The fact that $A_{i j}$ is negative corresponds to a relative velocity $v_{i j}^{-}$positive: the contact was opening at the beginning of the time step. Now, considering (9), two possibilities present themselves to us:

\section{the contact keeps opening if}

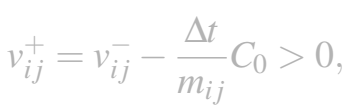

or, the contact starts closing if
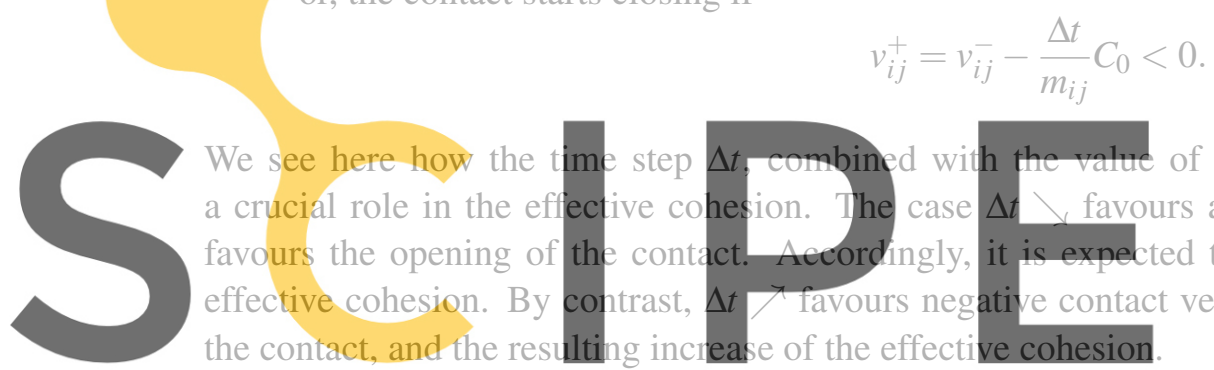

We see here how the time step a crucial role in the effecti favours the opening of th effective cohesion. By the contact, and the resulting
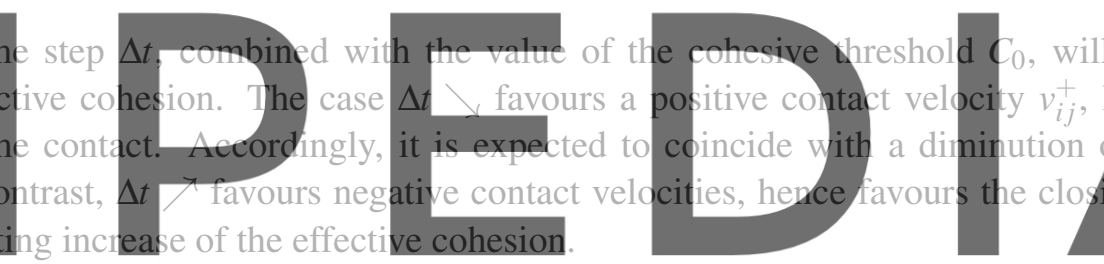

We use here the term effective cohesion as it works on favouring the closing or the opening of contacts,

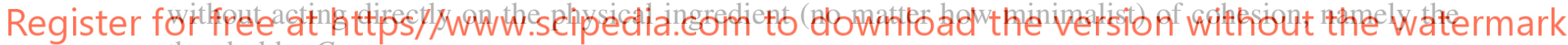
threshold $-C_{0}$.

Numerical simulations are now performed, considering complex packings rather than plain two-body systems, and we examine how the prediction steming from the simplest equations manifests itself in many-body systems.

\section{VERIFICATION: SIMULATING COHESIVE PILES}

\subsection{Simulations configuration}

To check how the predictions deduced from analysing the simple case of a binary collision show up in a more "realistic" complex system, we carry out CD simulations in two dimensions. Therefore, we consider a simple cohesive 150-grains-pile forming a step allowed to flow onto a rough horizontal plane (Figure 3). The grains are circular, showing a slight disparity in size, with diameters varying in the intervalle $[d-20 \%, d+20 \%]$, and a mean diameter $d=0.005 \mathrm{~m}$. The initial vertical and lateral dimensions of the pile are $H_{0} \simeq 10 d$ and $R_{0}=16 d$.

The grains interact through collisions with a coefficient of restitution $\rho=0.1$. Although friction was 
dropped from the equations developed in section 2 for the sake of simplicity, the Coulombic friction coefficient $\mu=0.5$ controls the value of tangential forces at contacts. In the following, $\rho$ and $\mu$ are kept constant.

The cohesive force threshold $C_{0}$ and the value of the computational time step $\Delta t$ were both varied. Following the literature, based on an analogy with fluid mechanics [3], we introduce the granular $B_{\text {ond }}$ number: the cohesive threshold $-C_{0}$ scales like the mean weight of the grains involved in the contact and the $B_{\text {ond }}$ number [10]:

$$
C_{0}=B_{\text {ond }} \times m_{i j} g
$$

with $m_{i j}=2 /\left(\frac{1}{m_{i}}+\frac{1}{m_{j}}\right)$.

Based on a previous stability analysis for larger systems [11], the initial height of the pile $H_{0} \simeq 10 d$ is expected to coincide with a stable state for $20 \lesssim B_{\text {ond }}$; this is indeed what we observe.

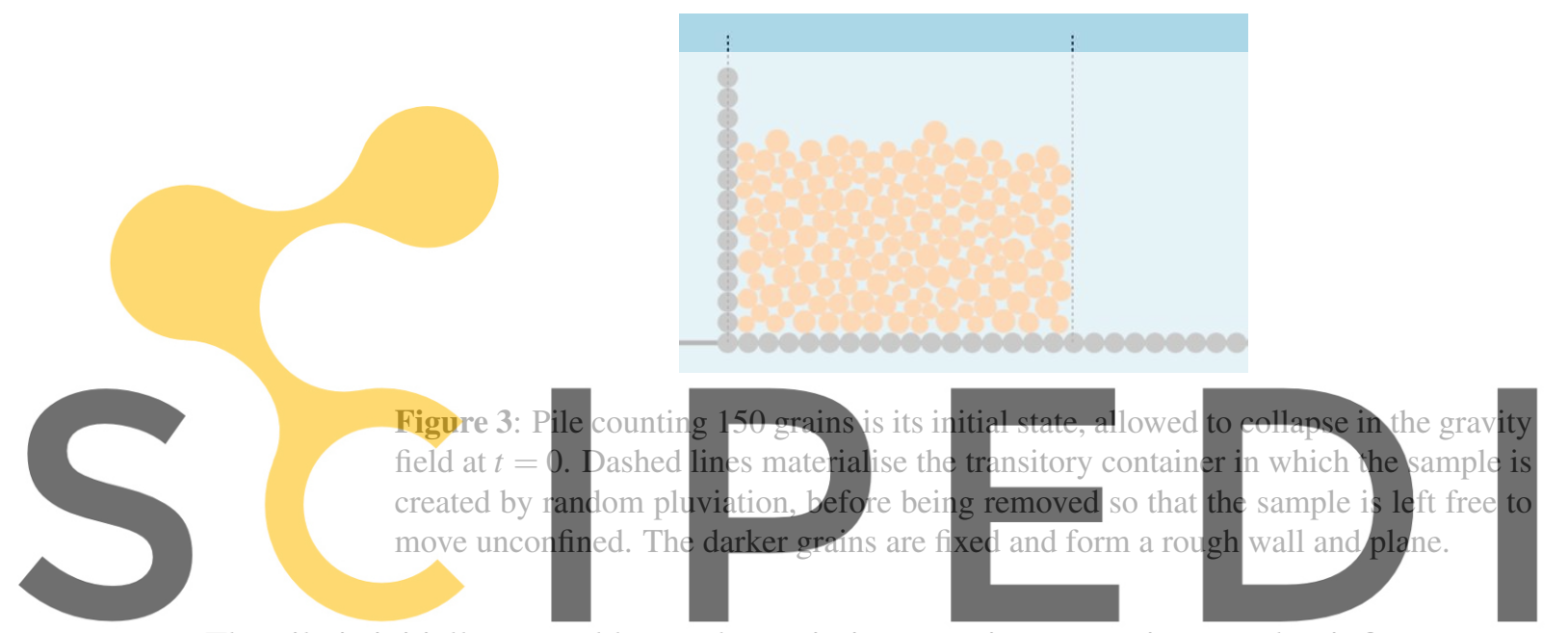

The pile is initially created by random rain in a transitory container, so that it forms a rectangular pile,

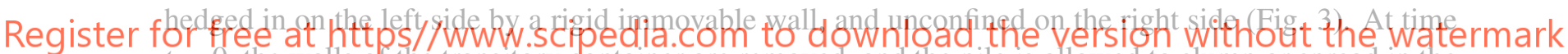 $t=0$, the walls of the transitory container are removed, and the pile is allowed to slump or spread in the}

gravity field (as in $[11,12]$ ).

We perform a series of 20 simulations using the exact same initial state, only varying the cohesion threshold $-C_{0}$ and the time step $\Delta t$ for performing the simulation run.

The cohesion threshold is varied through changing the bond number $B_{\text {ond }}$, set alternatively to $B_{\text {ond }}=0$, 5, 10, 15 and 18. The computational time step $\Delta t$ was alternatively set to $\Delta t=1 \cdot 10^{-4} s, 2.10^{-4} s$, $5.10^{-4} s, 7.10^{-4} s$ and $1.10^{-3} s$, the characteristic physical time scale for the particles dynamics being $\sqrt{d / g} \simeq 0.022 s\left(g=9.8 m \cdot s^{-2}\right)$. The physical duration of each collapse was set to $1 \mathrm{~s}$, irrespective of $B_{\text {ond }}$ and $\Delta t$.

The final states for 16 combinations of $\left(B_{\text {ond }}, \Delta t\right)$ are shown in Fig. 4. We observe that depending on the $B_{\text {ond }}$ number and $\Delta t$, the pile spreads out or retains a shape comparable with the initial state. These differences are discussed in the following. 


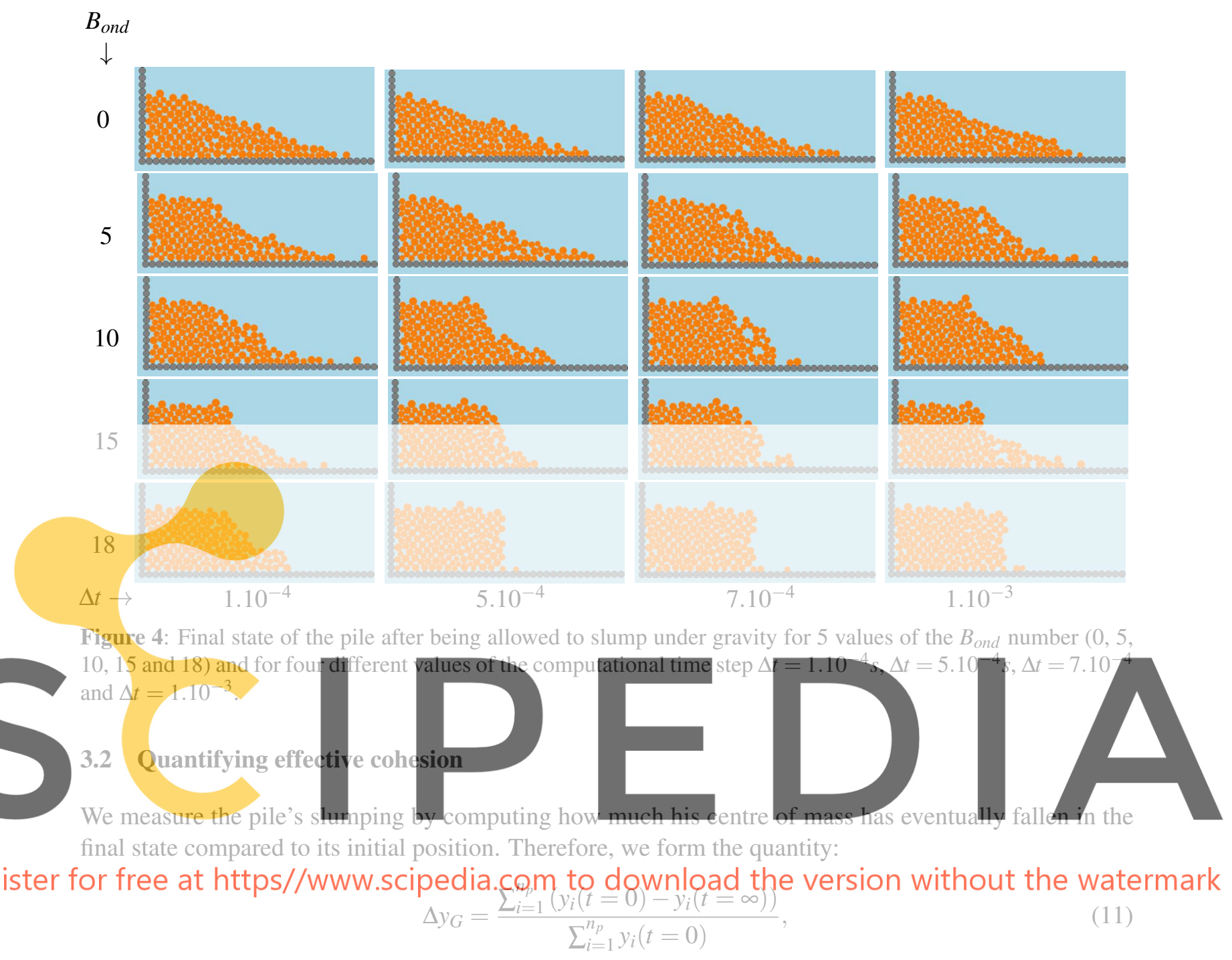

where $n_{p}$ is the number of grains (150 here). For a very cohesive pile, $\Delta y_{G}$ will be zero or very small, while it will be maximum for cohesion-less systems.

The vertical slump $\Delta y_{G}$ is plotted in Fig.5 as a function of the value of the computational time step $\Delta t$ used for performing the simulations. The case $B_{\text {ond }}=0$, namely cohesion-less contacts, gives us an insight in the unpredictable role of the duration of the time step $\Delta t$. Allowing larger $\Delta t$ tends to favour larger displacements, but the effect on the overall shape of the final grains packing is not straightforward. It is very dependent on the details of the initial arrangement and size distribution of the grains, and will inherently vary from one system to the other.

The results presented here, from the analysis of the behaviour of one single packing, are an illustration of what is likely to happen, but are no definitive answer. Therefore, an analysis with a representative number of independent systems is necessary, with corresponding error bars [13]. 


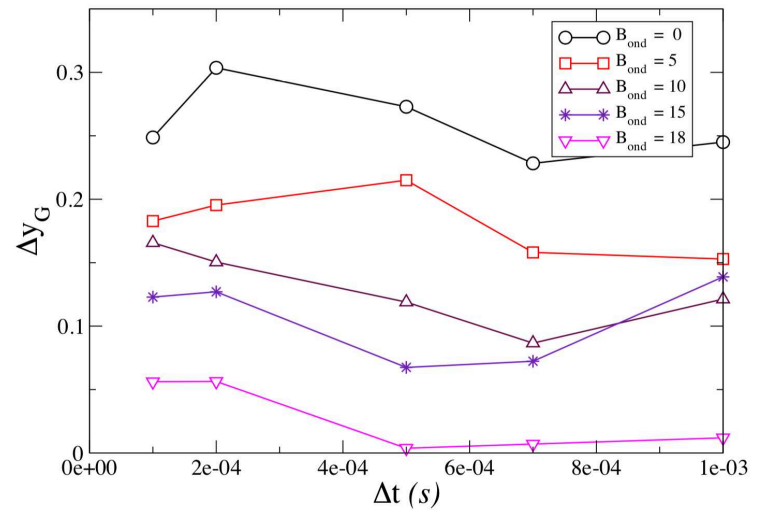

Figure 5: Vertical slump $\Delta y_{G}$ (equation (11)) as a fonction of the computational time step $\Delta t$ for 5 values of the $B$ ond number.

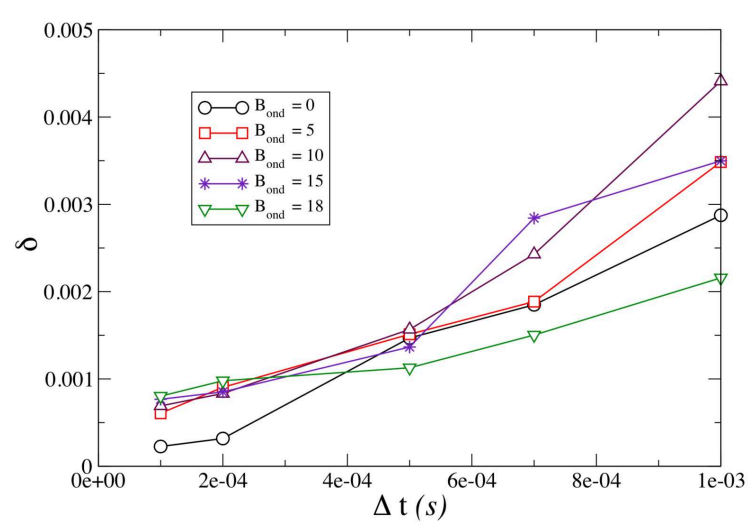

Figure 6: Mean overlap $\delta$ at the contacts as a function of the computational time step $\Delta t$ for all runs $\left(0 \leq B_{\text {ond }} \leq\right.$ 18).

Nevertheless, we see how the non-cohesive systems $B_{\text {ond }}=0$ may rearrange differently depending on $\Delta t$. The same variability due to increasing $\Delta t$ is expected to occur when contacts become cohesive, though in a lesser extent, and challenged by the action of cohesion forces. Consistently, the case $B_{\text {ond }}=5$, displaying smaller values of the vertical slump $\Delta y_{G}$ as expected for cohesive systems, also shows similar variations with $\Delta t$, but of a smaller amplitude than the case $B_{\text {ond }}=0$ (Fig. 5).

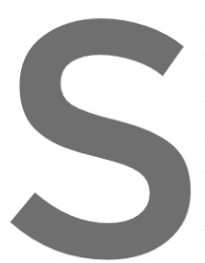
Increasing the cohesion to like, not shown), we observe the cohesion with $\Delta t$ as predicted by equation (9), best Yet the evolution is non-monotonous for larger $B$ for $B_{\text {ond }}=10$, or $\Delta t>5 \cdot 0^{-4}$ for $B_{\text {ond }} \geq 15$. In o thr
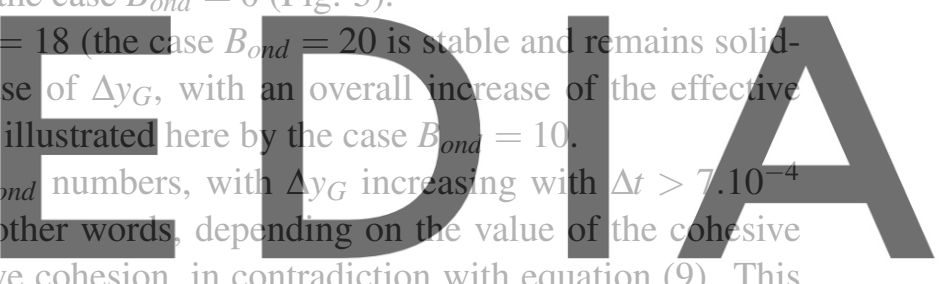
threshold $-C_{0}$, increasing $\Delta t$ decreases the effective cohesion, in contradiction with equation (9). This

\subsection{Precision and effective cohesion}

The tendency of the effective cohesion to decrease with $\Delta t$ for larger values of the latter is partly explained in Fig.6, showing the mean normalised overlap $\delta$ at contacts over the duration of the simulations as a function of the time step $\Delta t$ used for the simulations.

The mean normalised overlap $\delta$ is defined as the mean value of the sum of the radius of the two grains at contacts minus the distance between the two centres of mass:

$$
\delta=\frac{1}{n_{c}}\left(\sum_{\alpha=1}^{n_{c}} \delta_{\alpha}\right) / d=\left(\frac{1}{n_{c}} \sum_{\alpha=1}^{n_{c}} \frac{d_{i}+d_{j}}{2}-r_{i j}^{\alpha}\right) / d,
$$

where $n_{c}$ is the number of contacts, $i$ and $j$ are the two particles involved in the contact $\alpha$ and $r_{i j}^{\alpha}$ the distance between their centre of mass, and $d$ the mean grain diameter, computed over the duration of the simulation. Ideally, a contact $\alpha$ between two perfectly hard grains corresponds to $\delta_{\alpha}=0$; an overlap at 
contact $\alpha$ corresponds to $\delta_{\alpha}>0$; the case $\delta_{\alpha}<0$ corresponds to no contact. In practice, $\delta_{\alpha}$ is always positive at a contact, but its value remains very small when the computation is precise.

We observe in Fig. 6 how $\delta$ increases with $\Delta t$, namely the hard-core approximation becomes more and more loosely observed ${ }^{1}$. The overlap slowly increases as the positions of the grains are updated before forces are recomputed and adapt the contact graphs. Each new time step, as the new positions of the grains are computed, the list of contacts, namely of grains touching (i.e. such that $\left(d_{i}+d_{j}\right) / 2-r_{i j}^{\alpha}$ is positive) is updated. At any time, if the relative velocity of the touching grains is positive (corresponding the contact's opening), the newly computed distance between the two grains increases. Hence, the greater $\Delta t$, the greater the distance between the two grains, and the contact might well be lost at the end of the time step. In that case, $\Delta t \nearrow$ coincides with a loss of cohesive contacts, then a decrease of the effective cohesion at the scale of a pile. Equivalently, $\Delta t \searrow$ will coincide with an increase of effective cohesion, contrarily to what is expected from equation (9). In this case, cohesive contacts are less cohesive, but they are preserved.

\section{INTRODUCING AN IMPULSION THRESHOLD RATHER THAN A FORCE THRESHOLD \\ 4.1 Equations}

As already discussed in section 2.2.3, the equation controlling the relative velocity of a contact having reached the cohesive threshold reads:
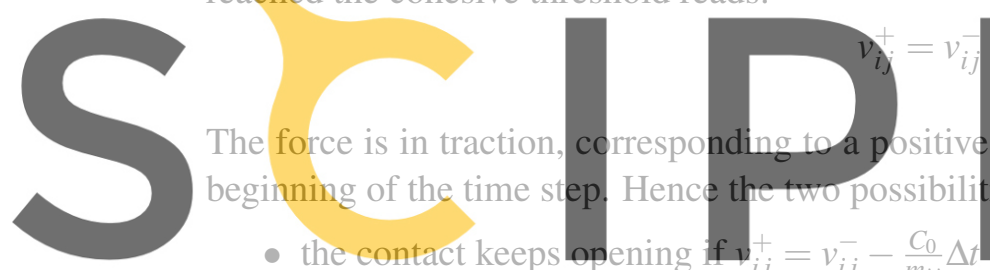
- the contact keeps opening if $\boldsymbol{v}_{i j}^{+}=v_{i j}^{-}-\frac{C_{0}}{m_{i j}} \Delta$
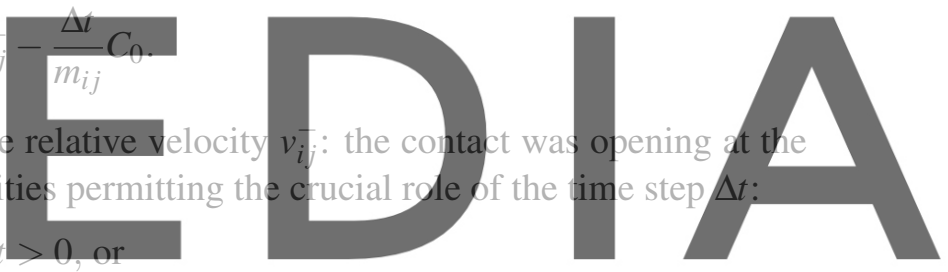

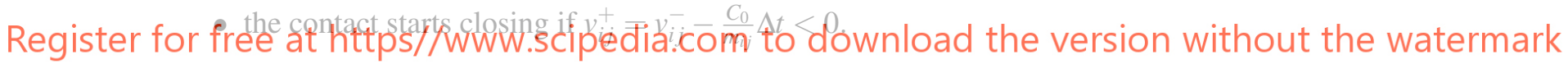
An easy un-sophisticated idea for fixing the dilemma is to consider the cohesive threshold in terms of impulse, rather than in terms of forces, writing instead:

$$
v_{i j}^{+}=v_{i j}^{-}-\frac{I_{0}}{m_{i j}},
$$

where $I_{0}=C_{0} \Delta t$ is constant. This means that the resistance of the contact is not set by a maximum force value, but by the integration of the force over the duration of the time step. Hence, we propose here to replace the cohesive force threshold $-C_{0}$ by the impulse threshold $I_{0}$. We introduce therefore a reference time duration $\Delta t_{r e f}$, which sets the reference value of the threshold. But then, if the time step $\Delta t$ is varied,

\footnotetext{
${ }^{1}$ For larger $\Delta t, \delta$ diverges and may exceed $1 \%$, but then the computation becomes of very poor quality, not shown here.
} 
the cohesive force must also be varied for the impulse threshold to remain constant:

$$
\begin{aligned}
& I_{0}=-B_{\text {ond }} \times m g \times \Delta t_{\text {ref }}=-C_{0} \Delta t_{\text {ref }} \\
& I_{0}=-B_{\text {ond }} \frac{\Delta t_{\text {ref }}}{\Delta t} \times m g \times \Delta t=-C_{0}^{\prime} \Delta t \\
& I_{0}=-B_{\text {ond }}^{\prime} \times m g \times \Delta t=-C_{0}^{\prime} \Delta t,
\end{aligned}
$$

with $B_{\text {ond }}^{\prime}=B_{\text {ond }} \frac{\Delta t_{\text {ref }}}{\Delta t}$.

In other words, we have shifted the problem of unwillingly changing the effective cohesion when changing the time step, to having to change the physical cohesion when changing the time step. Although this hardly sounds a promising approach, the next paragraph shows in practice what results are obtained by introducing the impulse threshold $I_{0}$.
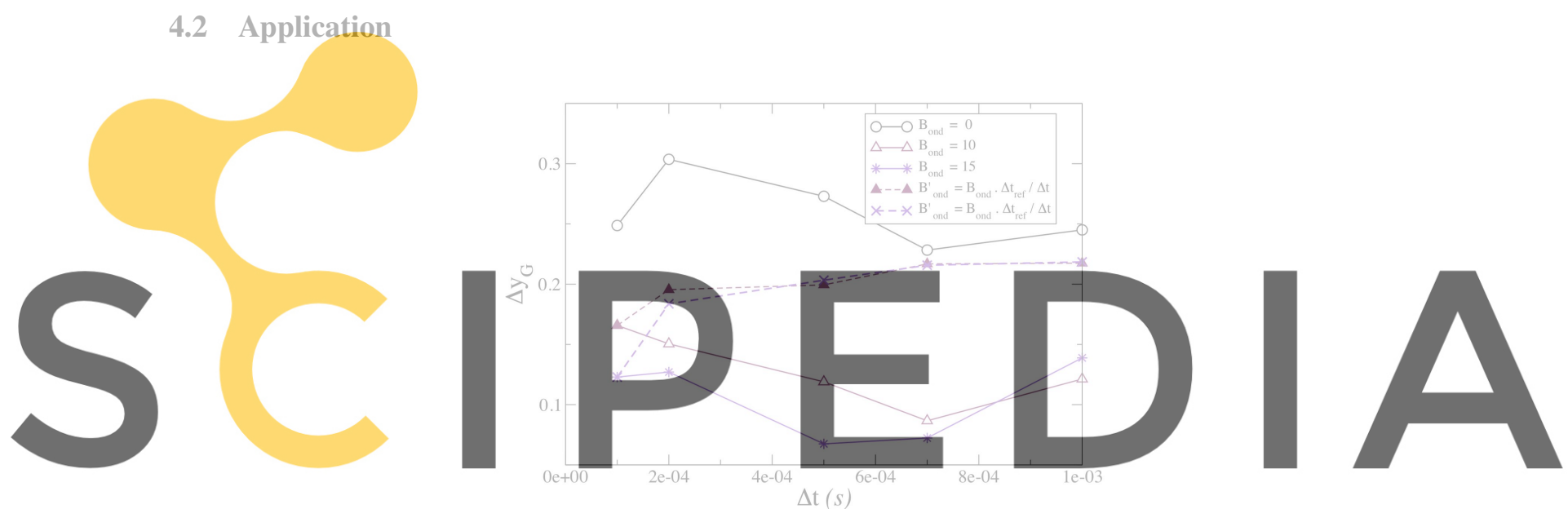

Register for free at https//www.scipedia.com to download the version without the watermark

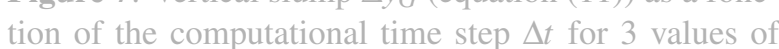

the $B_{\text {ond }}$ number, for a conesive threshold introduced in terms of force $\left(C_{0}\right)$ or in terms of impulsion $\left(I_{0}\right)$.

We consider a cohesive threshold set by the value of the impulse $I_{0}=-C_{0} \Delta t$. We choose arbitrarily a reference value for the time interval $\Delta t_{\text {ref }}=1.10^{-4} \mathrm{~s}$. We thus have $I_{0}=-B_{\text {ond }} m g \times \Delta t_{\text {ref }}$. Changing the time step to any $\Delta t \neq \Delta t_{\text {ref }}$, having $I_{0}$ constant implies $I_{0}=-B_{\text {ond }}^{\prime} m g \times \Delta t$, where $B_{\text {ond }}^{\prime}=B_{\text {ond }} \frac{\Delta t_{\text {ref }}}{\Delta t}$. For instance, considering the series where $B_{\text {ond }}=10$ (section 3), re-doing the slump experiment changing the time step $\Delta t$ (taking alternatively the values $1.10^{-4} s, 2.10^{-4} s, 5.10^{-4} s, 7.10^{-4} s$, and finally $1.10^{-3} s$ ) but with a constant $I_{0}$, means that we will change the $B_{\text {ond }}$ number alternatively to $B_{\text {ond }}^{\prime}=10, B_{\text {ond }}^{\prime}=5$, $B_{\text {ond }}^{\prime}=2, B_{\text {ond }}^{\prime}=3 / 2$ and $B_{\text {ond }}^{\prime}=1$.

The resulting slump $\Delta Y_{G}$, giving the relative vertical fall of the centre of mass of the packing (equation (11)), is shown in Fig 7, together with the slump obtained with a threshold expressed in terms of force rather than impulse (as already presented in section 3). The cases $B_{\text {ond }}=10$ and $B_{\text {ond }}=15$ are both revisited. The differences induced by varying the time step are definitely smoothed out; yet $\Delta Y_{G}$ 
remains significantly different from the reference case $\left(\Delta t=\Delta t_{\text {ref }}=1.10^{-4}\right)$; this is particularly true for $B_{\text {ond }}=15$.

\section{CONCLUSION}

In this paper, we are stressing a subtle point of the Contact Dynamics algorithm, which is the role of the value of the time step $\Delta t$. While DEM simulations rely on a small-scale description of the dynamics, making them much dependent on the models chosen for the grains microscopic deformation, the CD algorithm ignores the microscopic scale. In this way, it frees itself from the difficulty of describing a phenomenology (the physico-chemical details of the contact mechanism) which is not necessarily relevant at the scale of the system of grains. However, doing so, it introduces a kind of intermediate time scale in which all contacts are tackled as simultaneous phenomena. In that sense, the time step forms a sort of "supra" collision duration, and hence, play the role of a coarse-graining parameter [14]. The simulations presented here illustrate this effect with the slump of cohesive piles; a non cohesive system is also considered. However, larger systems (more grains) and more independent simulations should be performed to allow definite analysis and conclusions.

In a practical perspective, this paper aims at introducing the equations of CD mechanics in a nutshell, and give an easy illustration of the important features of the non-smooth mechanics backing up the solver. It also insists on an intriguing point, which is how a seemingly purely numerical quantity $(\Delta t)$ may end up being a non-trivial ingredient of the phenomenological behaviour of the simulated system. Hence, the importance of being always self consistent in one's choice of a time step.

\section{Acknowledgements}

The authors are happy to thank Olivier Pouliquen for his embarrassing questions. The first author warmly thanks Farhang Radjai for his unfailing availability and insights.

This work is part of the COPRINT project (http://coprint226940055. wordpress.com) supported by the ANR grant ANR-17-CE08-0017.

\section{REFERENCES}

[1] Alam M. \& S. Luding, Rheology of bidisperse granular mixtures via event-driven simulations, Journal of Fluid Mechanics 476, 69-103 (2003)

[2] Cundall P. A., O. D. L. Strack, A discrete numerical model for granular assemblies, Geotechnique, 29(1), 47- 65 (1979)

[3] Nase, S. T., Vargas, W. L., Abatan, A. \& McCarthy, J. J., Discrete characterization tools for cohesive granular material, Powder Technology 116, 214-223 (2001)

[4] Di Renzo A. \& F. P. Di Maio, Comparison of contact-force models for the simulation of collisions in DEM-based granular flow codes, Chemical Engineering Science 59-3, 525-541 (2004)

[5] Lommen S., D. Schott, G. Lodewijks, DEM speedup: Stiffness effects on behavior of bulk material, Particuology 12, 107-112 (2014) 
[6] Mandal S., M. Nicolas, O. Pouliquen, Insights into the rheology of cohesive granular media, Proceedings of the National Academy of Sciences, 117 (15), 8366-8373 (2020)

[7] Jean M. \& J.-J. Moreau, Unilaterality and dry friction in the dynamics of rigid bodies collections. In A. Curnier, (ed), Proc. of Contact Mech. Int. Symp., pp. 31-48 (1992)

[8] Moreau J.-J., Some numerical methods in Multibody Dynamics: Application to Granular Materials, European Journal of Mechanics, A/Solids 13-4, 93-114 (1994)

[9] Kadau D., Bartels G., Brendel L. \& D. E. Wolf, Contact dynamics simulations of compacting cohesive granular systems, Computer Physics Communications 147, 190-193 (2002)

[10] Rognon P. G. , J.-N. Roux, M. Naaïm \& F. Chevoir, Dense flows of cohesive granular materials, $J$. Fluid Mech. 596, 21-47, doi:10.1017/S0022112007009329 (2008)

[11] Abramian A., Staron L. \& P.-Y. Lagrée, The slumping of a cohesive granular column: Continuum and discrete modeling, Journal of Rheology 64 (5), 1227-1235 (2020)

[12] Staron, L., \& Hinch, E. , Study of the collapse of granular columns using two-dimensional discretegrain simulation, J. of Fluid Mech. 545 , 1-27, doi:10.1017/S0022112005006415 (2005)

[13] Staron L., A. Abramian \& P.-Y. Lagrée, Capturing the failure of a cohesive granular step, Proceeding of Powders\&Grains 2021, to appear

[14] Farhang Radjai, personal communication, 2020 